UVEITIS

\section{Uveitis and neurological diseases}

\section{K Gordon}

\section{Therapeutic and diagnostic challenges}

$\mathrm{S}$ ystemic disease associations in uveitis are widespread, ranging from inflammatory bowel disease (IBD), such as ulcerative colitis or Crohn's disease, to demyelinating diseases such as multiple sclerosis. ${ }^{1-3}$ In some cases, the systemic disease is diagnosed before onset of uveitis, whereas in others uveitis is the initial presentation of the underlying or associated condition. Two significant questions arise regarding neurological concomitants of uveitis. What is the incidence and identity of neurological diseases seen in individuals with uveitis? Are there particular clues in the clinical examination of an individual patient with uveitis that would help lead to diagnosing an associated neurological condition? In the current issue of BJO (p 1498), Smith and Rosenbaum attempt to address these important questions through a retrospective analysis of 1450 patients examined in a tertiary care uveitis clinic.

Increased use of biological agents such as the tumour necrosis factor $(\mathrm{TNF}-\alpha)$ inhibitors intensifies the relevance of this study. Targeted immunotherapy for specific immune mediated diseases is rapidly advancing. The immune tolerance network (ITN) is an international consortium with investigators from more than nine countries with the goal of developing clinical therapies for tolerance induction in transplantation, allergy and asthma, and autoimmune diseases. ${ }^{4}$ In contrast with the goals of tolerance, clinical trials in cancer therapy now include methods for boosting antitumour immunity through autologous dendritic cell delivery of recombinant tumour antigens or through inhibition of the negative regulatory $\mathrm{T}$ lymphocytes. ${ }^{56}$ This is an exciting time in therapeutic development targeted towards immune regulation. However, unanticipated responses to these therapeutic agents provide us with clues about the unknown aspects of disease pathogenesis. For example, use of the TNF- $\alpha$ inhibitors, whose positive therapeutic utility was predicted for multiple sclerosis, may actually increase demyelinating events. ${ }^{7}$

Use of biological agents in uveitis therapy has received increasing attention and there is now evidence for the utility of specific agents in the therapy of refractory uveitis or for specific uveitis syndromes-for example, Behçet's disease. ${ }^{9}$ However, therapy using an anti-TNF- $\alpha$ medication should probably not be initiated in a patient with underlying multiple sclerosis. Additionally, immunosuppressive medications, commonly used in treatment of refractory uveitis, may mask or worsen certain infectious or neoplastic disease concomitants of uveitis. Understanding the magnitude of the neurological associates of uveitis and developing preferred patterns for the comprehensive evaluation of patients with specific uveitis syndromes is critical in this emerging therapeutic era.

In the series presented here by Smith and Rosenbaum, almost $8 \%$ of all patients in their clinic had neurological disease underlying and associated with the ocular inflammatory disease. The authors note, but do not describe, cases with neurological diseases that were presumably unrelated to uveitis. These cases were excluded from the present report; however, additional descriptions of these patients may have been helpful to understanding the magnitude of neurological diseases in this patient group. For example, associations of HIV infection with both infectious diseases and uveitis are well characterised. $^{10}{ }^{11}$ The absence of AIDS or HIV as a diagnosis in the present series probably reflects the exclusion of these patients from this report.

Future prospective studies will provide the basis for developing evidence based indications for both evaluation and therapy of these important diseases

It is notable, but not unexpected, that specific clinical features of uveitis were helpful in diagnosing Vogt-KoyanagiHarada (VKH), multiple sclerosis, and herpes virus infections. However, the sensitivity of the clinical examination in making the correct systemic diagnosis was insufficient in 25\% of patients with MS and $20 \%$ of patients with herpes infections. These numbers are of significant concern as the therapeutic decision making would have great potential negative impact on the underlying disease. Furthermore, 24\% of patients in this series with both uveitis and a neurological disease did not have a specific neurological diagnosis, further complicating the therapeutic decision tree.

The authors freely note some of the additional limitations of their study. These limitations include the retrospective nature of the study, the possibility of underestimating the prevalence of diagnosing concomitant neurological diseases, and the changing neurological disease definitions during the time in which patients were examined (19852000). One important potential limitation of any retrospective analysis includes the lack of long term follow up data-for example, a patient who is successfully treated for uveitis may subsequently develop an associated demyelinating disease but may not be re-evaluated by the uveitis specialist unless the ocular inflammation recurs. Additional potential limitations include the applicability of data from a tertiary care facility to the larger experience in community treated uveitis patients. These limitations do not detract from the study by Smith and Rosenbaum, but rather suggest additional prospective studies are required to address the magnitude and scope of neurological diseases in patients with uveitis.

How should the present study be used in clinical practice? Awareness of the underlying, uveitis associated neurological diseases should be increased among both comprehensive ophthalmologists and rheumatologists who deliver care to many of these patients. In evaluating patients with uveitis, a comprehensive history must be performed to elicit symptoms that may suggest systemic conditions such as multiple sclerosis, which may initially have intermittent, self limited systemic complaints not typically reported to the ophthalmologist. Certainly, before initiation of anti-TNF- $\alpha$ therapy, demyelinating diseases such as multiple sclerosis, with a prevalence of at least $1 \%$ in this series, must be considered. The role for additional diagnostic testing, such as neuroradiological imaging, analysis of spinal fluid, or sampling and pathological evaluation of intraocular fluids must be individually considered for each patient. Importantly, future prospective studies, which define the prevalence and characteristics of specific neurological syndromes in association with uveitis, will provide the basis for developing evidence based indications for both evaluation and therapy of these important diseases. 
Br J Ophthalmol 2004;88:1483-1484.

Correspondence to: Lynn K Gordon, Jules Stein Eye Institute, 100 Stein Plaza, Los Angeles, CA 90095, USA; Igordon@ucla.edu

\section{REFERENCES}

1 Dunn JP, Nozik RA. Uveitis: role of the physician in treating systemic causes. Geriatrics 1994:49:27-32.

2 Smith JR, Coster DJ. Diagnosing the systemic associations of anterior uveitis. Aust N Z J Ophthalmol 1998;26:319-26.
3 Cooper GS, Stroehla BC. The epidemiology of autoimmune diseases. Autoimmun Rev 2003:2:119-25.

4 Rotrosen D, Matthews JB, Bluestone JA. The immune tolerance network: a new paradigm for developing tolerance-inducing therapies. J Allergy Clin Immunol 2002;1 10:17-23.

5 O'Neill DW, Adams S, Bhardwaj N Manipulating dendritic cell biology for the active immunotherapy of cancer. Blood 2004; (in press).

6 Schultes BC, Nicodemus CF. Using antibodies in tumour immunotherapy. Expert Opin Biol Ther 2004:4: 1265-84.

7 Wiendl H, Hohlfeld R. Therapeutic approaches in multiple sclerosis: lessons from failed and interrupted treatment trials. BioDrugs 2002; 16: 183-200.
8 Nussenblatt RB. Bench to bedside: new approaches to the immunotherapy of uveitic disease. Int Rev Immunol 2002;21:273-89.

9 Greiner K, Murphy CC, Willermain F, et al. AntiTNFalpha therapy modulates the phenotype of peripheral blood CD4+ T cells in patients with posterior segment intraocular inflammation. Invest Ophthalmol Vis Sci 2004;45:170-6.

10 Nussenblatt RB, Lane HC. Human immunodeficiency virus disease: changing patterns of intraocular inflammation. Am J Ophthalmol 1998;125:374-82.

11 Levinson RD, Vann R, Davis JL, et al. Chronic multifocal retinal infiltrates in patients infected with human immunodeficiency virus. Am J Ophthalmol 1998;125:312-24.

ROP

\section{Threshold ROP}

\section{Clark}

\section{Where next?}

$\mathrm{R}$ etinopathy of prematurity (ROP) remains a major cause of visual loss in very premature infants. First described by Terry ${ }^{1}$ in 1942 the condition reached epidemic proportions before the introduction of controlled oxygen administration. During the 1970 s and 1980s a second epidemic occurred as a result of the increased survival of very low birthweight infants (under $1000 \mathrm{~g}$ ).

While the incidence and severity of ROP have reportedly declined in some centres, there still remains a significant threat to vision in infants born between 23 and 26 weeks gestation. ${ }^{2}$ The retinopathy progresses more rapidly in this group and may proceed directly from immature vessels without the appearance of the characteristic ridge. The choroidal vessels are readily visible through the pale retinal pigment epithelium leading to the false interpretation that the retinal vasculature is more fully developed ${ }^{3}$

The increased survival of these infants and their subsequent transfer from regional back to more local neonatal units may mean ophthalmologists with limited experience being requested to examine and interpret the atypical features of retinopathy associated with these babies.

In this issue of the BJO (p 1500), Schulenburg and Tsanaktsidis demonstrate photographically and by fluorescein angiography the morphologically different ROP often seen in infants born less than or equal to 25 weeks gestational age. Applying the conventional threshold for treatment in these cases may lead to a poor outcome, whereas recognition of this different morphology and its speed of progression is fundamental to successful management.

The functional outcome at 10 years of age of threshold cryotherapy treated infants was disappointing. Distance acuity in the range $6 / 60$ to $6 / 18$ occurred in $30.4 \%$, and only $25.2 \%$ had a visual acuity 6/12 or better. ${ }^{4}$ Laser treatment may offer advantages over cryotherapy and has been associated with better functional outcomes. ${ }^{5}$

Laser certainly makes treating posteriorly located ROP easier and this may have contributed to the improved structural results. ${ }^{6}$

Should posterior disease be treated earlier?

Recent investigation into the early treatment of high risk prethreshold
ROP has shown better functional outcomes at 9 months of age than conventional treatment at threshold. ${ }^{7}$ Schulenburg and Tsanaktsidis's paper is timely because early recognition of the serious nature of the high risk signs of this atypical ROP predominantly seen in extremely premature babies of 2325 weeks gestational age, combined with appropriate early intervention, should improve the visual outlook in these cases.

Br J Ophthalmol 2004;88:1484. doi: 10.1136/bjo.2004.051771

Correspondence to: D Clark, University Hospital Aintree, Department of Ophthalmology, Walton Hospital, Rice Lane, Liverpool L9 1AE L9 1AE, UK; david.clark@aht. nwest.nhs.uk

\section{REFERENCES}

1 Terry TL. Extreme prematurity and fibroblastic overgrowth of persistent vascular sheath behind each crystalline lens: I. Preliminary report. Am J Ophthalmol 1942;25:203-4

2 Allegaert K, deCoen K, Devlieger H. Threshold retinopathy at threshold viability: the EpiBel study Br J Ophthalmol 2004;88:239-42.

3 Recchia FM, Capone A. Contemporary understanding and management of retinopathy of prematurity. Retina 2004;24:283-292.

4 Cryotherapy for Retinopathy of Prematurity Cooperative Group. Multicenter Trial of Cryotherapy for retinopathy of prematurity: ophthalmological outcomes at 10 years. Arch Ophthalmol 2001;119:1110-18.

$5 \mathrm{Ng}$ EYJ, Conolly B, McNamara JA, et al. A comparison of laser photocoagulation with cryotherapy fro threshold retinopathy of prematurity at 10 years. Ophthalmology 2002;109:928-34.

6 Noonan CP, Clark DI. Trends in the management of stage 3 retinopahy of prematurity. Br J Ophthalmol 1996:80:278-81.

7 Early Treatment for Retinopathy of Prematurity Cooperative Group. Revised indications for the treatment of retinopathy of prematurity. Arch Ophthalmol 2003;121:1684-96. 
BJO

\section{Found in translation}

\section{A H K Kwok, D S Pereira, R B Bhisitkul}

\author{
Introducing BJO in Translation
}

E nglish, as we at the BJO would be the first to point out, is a perfectly splendid language, but it does have one drawback: roughly six billion people don't speak it. As a first step in addressing the non-anglophone sector of the ophthalmological world, BJO Online is introducing a new feature: BJO in Translation.

The language barrier has remained surprisingly impervious to advances in the medical journal model. Readers and contributors from around the world still accommodate themselves to the hegemonic languages of the major journals. This was understandable in the predigital age, when the printing and distribution costs of the traditional print medical journal precluded the publication of multiple versions in multiple languages. But with the wide adoption of electronic publication over the past decade, such considerations have been obviated. Page limitations are meaningless in cyberspace, added costs of paper and printing are now just a matter of added megabytes, and fast distribution is available to any place on the planet with internet access. The opportunity is at hand for our new translation project.

BJO in Translation now features editions in Chinese and Portuguese. Each month, the abstracts for all of the issue's articles in clinical science and laboratory science are available in translation on the Chinese page and the Portuguese page of BJO Online. Online access to the abstract translation pages is free of charge; the main points of the article can be read in the Chinese and Portuguese abstracts and links to the full text English article are then available to subscribers.

\section{Jornal Britânico de Oftalmologia 英国眼科杂志}

The BJO in Translation Chinese edition is edited by Alvin Kwok in Hong Kong. Chinese is the most widely spoken language in the world, with approximately a billion speakers using the leading dialects. The BJO Chinese page uses the simplified version of traditional Chinese characters developed in China's 20th century language reform, which is the official text used by speakers of Mandarin, Cantonese, Hakka, and other dialects. There are an estimated 22000 ophthalmologists in China, with only a minority fluent in English. Most Chinese ophthalmologists are trained in a "Western medicine" educational system, but others are trained in schools of traditional Chinese medicine. Developing countries in Asia, like China, have a large load of preventable blindness. In a country of 9.56 million square kilometres with a population of 1.2 billion, nine million people in China have cataract blindness. To keep abreast of advances necessary to address the eye care needs of this huge population, a small portion of ophthalmologists are able to go abroad to receive training or attend conferences. But for the vast majority without this option, peer reviewed ophthalmological journals represent an important means to keep updated on advances in the field. Journals available in libraries have been integral in enhancing the practice of ophthalmology in China for the benefit of patients.

The BJO in Translation Portuguese edition is edited by Daniel de Souza Pereira and Jonathan Lake in São Paulo, Brazil. Portuguese is the eighth most widely spoken language in the world, with between 190 million and 210 million speakers in Portugal, Brazil, Angola, Mozambique, Cabo Verde, Guinea-Bissau, and São Tomé and Príncipe islands. From its Romance origins Portuguese reflects numerous linguistic influences including Arabic, Galician, Castilian, and French, giving rise to considerable variations in pronunciation and structure across its wide, non-contiguous geographic scope. Nevertheless, the language is mutually comprehensible across all regions where it is spoken, so despite the distinctly Brazilian accent of the BJO Portuguese edition, it targets readers in Portugal and other countries. The potential audience for a Portuguese version is significant; in Brazil alone there are approximately 11600 ophthalmologists, with 53 training programmes producing 360 new ophthalmologists per year.

The BJO in Translation continues our mission to emphasise the international scope of the British Journal of Ophthalmology, and to reaffirm the initiative of the BMJ family of journals to provide free online materials to readers and institutions in the developing world. It's our hope that the BJO in Translation will continue growing to incorporate other languages and expanded content. Only 6497 languages left to go.

Br J Ophthalmol 2004;88:1485. doi: $10.1136 /$ bjo.2004.057588

\section{Authors' affiliations}

A H K Kwok, Department of Ophthalmology, Hong Kong Sanatorium and Hospital, Hong Kong

D S Pereira, Santa Casa de São Paulo, Brazil R B Bhisitkul, UCSF Department of Ophthalmology 10 Kirkham Street, K301 San Francisco, CA 94143, USA

Correspondence to: Dr Robert B Bhisitkul, UCSF Department of Ophthalmology 10 Kirkham Street, K301 San Francisco, CA 94143, USA; bhisi@@itsa.ucsf.edu 